\title{
Evaluating the feasibility and impact of case rate payment for recovery support navigator services: a mixed methods study
}

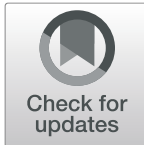

Maria E. Torres ${ }^{1,2^{*}}$, Mary Brolin ${ }^{1}$, Lee Panas ${ }^{1}$, Grant Ritter $^{1}$, Dominic Hodgkin ${ }^{1}$, Margaret Lee ${ }^{1}$, Elizabeth Merrick${ }^{1}$, Constance Horgan ${ }^{1}$, Jonna C. Hopwood ${ }^{3}$, Andrea Gewirtz ${ }^{3}$, Natasha De Marco ${ }^{4}$ and Nancy Lane ${ }^{1}$

\begin{abstract}
Background: Acute 24-h detoxification services (detox) are necessary but insufficient for many individuals working towards long-term recovery from opiate, alcohol or other drug addiction. Longer engagement in substance use disorder (SUD) treatment can lead to better health outcomes and reductions in overall healthcare costs. Connecting individuals with post-detox SUD treatment and supportive services is a vital next step. Toward this end, the Massachusetts Medicaid program reimburses Community Support Program staff (CSPs) to facilitate these connections. CSP support services are typically paid on a units-of-service basis. As part of a larger study testing health care innovations, one large Medicaid insurer developed a new cadre of workers, called Recovery Support Navigators (RSNs). RSNs performed similar tasks to CSPs but received more extensive training and coaching and were paid an experimental case rate (a flat negotiated reimbursement). This sub-study evaluates the feasibility and impact of case rate payments for RSN services as compared to CSP services paid fee-for-service.
\end{abstract}

Methods: We analyzed claims data and RSN service data for a segment of the Massachusetts Medicaid population who had more than one detox admission in the last year and also engaged in post-discharge CSP or RSN services. Qualitative data from key informant interviews and Learning Collaboratives with CSPs and RSNs supplemented the findings.

Results: Clients receiving RSN services under the case rate utilized the service significantly longer than clients receiving CSP services under unit-based billing. This resulted in a lower average cost per member per month for RSN clients. However, when calculating total SUD treatment costs per member, RSN client costs were 50\% higher than CSP client costs. Provider organizations employing RSNs successfully implemented case rate billing. Benefits included allowing time for outreach efforts and training and coaching, activities not paid under the unit-based system. Yet, RSNs identified staffing and larger systems level challenges to consider when using a case rate payment model.

(Continued on next page)

\footnotetext{
* Correspondence: metorres@smith.edu

'Brandeis University, Heller School for Social Policy and Management,

Waltham, MA 02453, USA

${ }^{2}$ Smith College School for Social Work, Lilly Hall, Northampton, MA 01060,

USA

Full list of author information is available at the end of the article
}

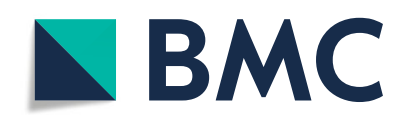

(- The Author(s). 2020 Open Access This article is licensed under a Creative Commons Attribution 4.0 International License, which permits use, sharing, adaptation, distribution and reproduction in any medium or format, as long as you give appropriate credit to the original author(s) and the source, provide a link to the Creative Commons licence, and indicate if changes were made. The images or other third party material in this article are included in the article's Creative Commons licence, unless indicated otherwise in a credit line to the material. If material is not included in the article's Creative Commons licence and your intended use is not permitted by statutory regulation or exceeds the permitted use, you will need to obtain permission directly from the copyright holder. To view a copy of this licence, visit http://creativecommons.org/licenses/by/4.0/. The Creative Commons Public Domain Dedication waiver (http://creativecommons.org/publicdomain/zero/1.0/) applies to the data made available in this article, unless otherwise stated in a credit line to the data. 
(Continued from previous page)

Conclusions: Addiction is a chronic disease that requires long-term investments. Case rate billing offers a promising option for payers and providers as it promotes continued engagement with service providers. To fully realize the benefits of case rate billing, however, larger systems level changes are needed.

Keywords: Substance addiction, Patient navigation, Third-party payers, Mental health recovery, Patient engagement, Payment reform, Case rate, Detoxification, Recovery support

\section{Background}

A high percentage of people with substance use disorders (SUD) experience more than one detoxification (detox) admission within a 12-month period [1-5]. Detox services at freestanding detox centers in Massachusetts provide medically monitored inpatient detoxification. Massachusetts data on detox admissions from 1996 to 2002 found individuals using detox services averaged five detox admissions a year [3]. More recent data from the Massachusetts Behavioral Health Partnership (MBHP), a Beacon Health Options company which manages behavioral health for many Massachusetts Medicaid enrollees, found that $57.7 \%$ of those receiving detox services in FY2011 had two or more admissions that year, and that this group of "repeaters" accounted for $87 \%$ of all detox admissions that year [6]. Repeated admissions to detox without engagement in follow-up care represent lost opportunities for recovery, highlight inadequate access to a limited resource, and come at significant human and financial costs $[5,7,8]$.

Successfully navigating the transition from detox to SUD treatment is crucial. Yet, continuity of care studies indicate that in most settings less than half of clients utilize post-detox SUD treatment within 14 days of discharge [9]. Various states fund services to support postdetox linkage. For example, Massachusetts offers Medicaid-billable community support program (CSP) services upon discharge from a detox. CSP offers support services to help consumers at high risk of relapse to access and use community-based behavioral health services [10]. Through their employer, CSP workers receive annual training on such topics as engagement and outreach, service coordination and principles of recovery; each employer determines which trainings will be offered [10]. CSP services are billed in 15-min units of service and require prior authorization from the insurer. Additionally, clients must meet medical necessity criteria. Strict criteria detail the range of services CSP workers can provide, which includes in-person or telephone case management, direct time with clients and providers, and travel, but not outreach services [11].

Despite patients' access to CSPs, data from MBHP indicates that only $30 \%$ of eligible clients see a CSP upon discharge from detox. Key factors that may contribute to low engagement include issues with the payment model and staffing. SUD treatment typically pays fee-for-service (FFS), which has both strengths and weaknesses. FFS payments incentivize more care and provide higher payments for clients needing more care [7, 12, 13]. FFS payments do not reward quality care and restrict the scope of services covered (e.g. outreach not covered) [12]. Given the complex needs of high-frequency users of detox services, these drawbacks are significant.

Case rate payment, may offer a better option than FFS payment to reimburse care navigator services for patients with repeated detox admissions. Under a case rate payment, providers receive a previously negotiated flatrate for services delivered over a certain period [14]. Case rate payment offers a cost-effective way to pay for services for individuals with complex needs $[12,13,15]$. A case rate can allow RSN workers to have greater flexibility in their work with clients, allowing more outreach and follow up and, with no penalty to their income, they might participate in more training and professional development, improving quality of care. Moreover, a case rate system reduces administrative burden for both providers and payers as it does not require the same level of tracking and billing infrastructure.

Case rate billing has not been tested for RSN or CSP services among those with repeated admissions to detox. As a component of a larger study, this substudy addressed this gap, assessing the feasibility and impact of case rate payment for RSN services using the Medical Research Council (MRC) framework for the evaluation of complex interventions [16]. This framework, provides guidance on process evaluation of interventions that contain many interacting components, particularly when the key aim of the evaluation is to determine whether it is effective in everyday practice [17]. As such, the MRC framework focuses on assessing the overall implementation and impact of interventions taking into consideration contextual factors [16]. The overarching research question that guided this study asked: Is case-rate payment for RSN services a feasible alternative to unit-of-service billing currently in place for CSP services for clients with repeated admissions to detox within a 12-month period? Feasibility can be determined using many different criteria including effects on subsequent detox admissions or overall health care costs. Those criteria are 
described elsewhere [18]. For this component of the study, feasibility was determined based on the following criteria: (1) Did case rate billing provide an economical alternative to FFS billing for this population? (2) Did the case rate payment method improve client length of engagement for clients receiving RSN services compared to clients receiving CSP services paid FFS? and (3) How did advantages and disadvantages of the case rate payment method influence providerlevel practices?

\section{Methods}

\section{Study setting}

This mixed methods study took place from March 2013 through March 2015, under a grant from the Centers for Medicare and Medicaid Services (CMS) to MBHP, which manages behavioral health services for a portion of the MassHealth (Massachusetts Medicaid) Program. Brandeis University was MBHP's academic partner. All detox providers funded by MBHP were invited to participate in the study. Site visits to describe the study and address any questions about participation were made to all invited programs. There was no consequence for nonparticipation. Of the 14 provider organizations invited, 13 choose to participate. The one provider that declined the invitation to participate did not provide a reason for their decision. This provider served the same population as participating programs and was not substantively different from participating programs in terms of location, criteria for admission, or services provided. Detox providers willing to join the study were assigned to either Treatment-As-Usual (TAU, nine sites) or Intervention (four sites) group based on client volume. Our aim was to minimize the number of providers offering the intervention to allow the research team to closely monitor implementation. Data from the year before the study (FY2011) indicated that the four intervention providers covered $54 \%$ of the detox units of service that year [6]. Providers in both groups represented urban and suburban settings and larger and smaller programs.

Within the study, RSNs performed similar tasks to CSPs but received more extensive training and coaching and were paid via the experimental case rate. TAU detox programs continued to employ CSP workers. At intervention detox sites, CSP workers were trained to become Recovery Support Navigators (RSNs). As needed, intervention sites also hired new staff who were trained as RSNs. RSNs provided CSP-like services with a few notable differences. All RSNs completed an initial training and participated in monthly in-person coaching sessions and quarterly trainings as part of a larger study, described elsewhere [19]. Organizations providing RSN services were paid a daily case rate for each client on RSNs' caseloads, and RSNs had no restrictions on the type or volume of services they provided to clients. However, RSNs needed to meet/connect with each client at least once within a 30-day period to qualify for continued payment. To capture the type and volume of services provided, RSNs tracked the nature, type and frequency of each client contact via a weekly RSN service $\log$.

\section{Data sources}

Quantitative data came from MBHP Medicaid claims data and RSN service data for RSN clients. For CSP clients, the only service delivery data captured were CSPs' requests for service authorization.

Qualitative data were collected by the two lead authors and a graduate research assistant, who are trained in qualitative methods, using key informant interviews and detailed meeting notes from RSN and CSP Learning Collaboratives (LCs). Research staff conducted ten onehour in-person interviews with RSN staff at each of the four intervention sites during the second year of the study to develop a more in-depth understanding of the training and support needed by RSNs, the benefits and challenges of offering RSN services and the impact of other aspects of the intervention on their work. Seven interviews with RSNs and three interviews with RSN supervisors were completed. The interviewers did not have relationships with the provider staff prior to the study, but at the study's outset they shared the purpose and goals of the study with provider staff. The qualitative research team invited the RSN supervisors and key RSN staff, those whose caseloads were predominantly RSN cases, to take part in the interviews. In total, only one RSN supervisor was not available for the interview. All others agreed to take part in the interview. Interviewees signed a consent form prior to the interview. There was no financial compensation for those who chose to participate. Interviewers used an interview guide, took notes and requested permission to audio record the interviews. The research team wrote detailed notes from the audio recordings and coded them with their field notes to summarize and identify themes. LCs were held at MBHP's offices when in-person (10 meetings) and by telephone (4 meetings) and conducted in the second and third years of the study. LC participants included representatives from the evaluation team and key staff from participating RSN and CSP providers. Fourteen 90-min LCs were held, seven for intervention providers (11 attendees) and seven for TAU providers (12 attendees). Themes discussed in the LC included questions about barriers and facilitators for clients who had access to RSN or CSP services, service delivery challenges, as well as questions about other aspects of RSN and CSP service delivery. The meetings had formal agendas developed by the research staff, who took detailed notes and wrote 
individual meeting summaries and annual summaries. One to three representatives from MBHP were sometimes present at the LC to answer study related questions, present preliminary claims data, or address RSN or CSP service delivery questions or concerns. The key informant interviews and LCs offered opportunities to learn about implementation successes and challenges.

\section{Participant eligibility criteria and recruitment}

Study participants were members of the MassHealth Primary Care Clinician (PCC) plan aged 18-64 whose behavioral health care was managed by MBHP. Plan members became study eligible if (1) they were admitted to a detox program and had received authorization for a detox admission at least once in the prior 12-month period, (2) this "index admission" took place between March 29, 2013 and March 31, 2015, and (3) they lived within the respective TAU or intervention provider catchment area. Members choosing to receive services were classified between the RSN and CSP analytic samples based on the location of their index admission (intervention or TAU). The study protocol was reviewed and approved by the New England Institutional Review Board.

\section{Study measures}

Study variables included client gender, age, whether there was any prior detox within 90 days, Charlson Comorbidity Score [20], mental health diagnosis, Medicaid enrollment category, and length of Medicaid enrollment. ICD-9 codes in the claims data were used to determine whether a comorbid mental health diagnosis was present, and to construct the Charlson Comorbidity Score for number of chronic conditions. This index is computed by counting how many comorbid illnesses a patient has from a pre-established list of 17 , and assigning weights greater than 1 to certain more severe illnesses [20].

CSP claims data were analyzed at both the person level and the service unit level (15-min units), while RSN data were analyzed at the person level and the case rate level (a daily rate paid in monthly increments). Costs were reported on a per member and per member per month basis, to adjust for potential differences between case rate and unit level billing.

To determine total costs per member over the entire study, the number of units billed for CSP services and the number of days billed for RSN services post-index detox admission were identified, and multiplied by the corresponding payment rates. Per member per month costs were calculated using the number of units or days billed in the month multiplied by the corresponding payment rate. Length of engagement was calculated as days between the first and the last service day. Claims data included 816 RSN clients and 924 CSP clients. RSN service data included 799 clients; 17 clients were missing RSN service data.

\section{Analytic approach}

Descriptive statistics assessed differences between the larger study sample and the subset of clients who engaged in RSN or CSP services. For the larger study sample, eligible clients were assigned to a study arm based on intentionto-treat. Client assignment depended on the treatment program they were admitted to when they became eligible for the study, their "index" admission. Assignment was fixed for the length of the study, and was not dependent on the client's decision to accept RSN or CSP services. The intention-to-treat approach is less vulnerable to selection effects which could bias the analyses if they had been limited to only those subjects who accepted services. To answer the research questions, greater emphasis was placed on the quantitative data, using the qualitative data to provide context and nuance to the findings. Feasibility of the case rate intervention was assessed by comparing per member per month and total costs between CSP and RSN clients. Emphasis was given to the per member per month cost, underscoring the importance of longer engagement with services.

To determine how the case rate impacted service delivery, analyses focused on the type of contact and amount of time spent on each task. CSP staff cannot bill for outreach attempts, thus all CSP contact is defined as service contact. RSN service data $(n=799)$ differentiated the type of contact (outreach or service contact) and noted the amount of time spent on each task.

Qualitative data on the role and impact of providerlevel context were coded by the three qualitative interviewers, with themes derived from the data, to summarize, synthesize and sort the information for analysis. All data were double coded and differences between coders resolved. The resulting coding tree included three themes with seven categories or parent codes across the themes and twelve child codes across the categories. Framework analysis was used to identify key themes and to provide context to the quantitative analyses. This method has been used with increasing frequency in health services research to provide a structured means for reducing qualitative data while maintaining the context of data within and across interviews; it is both inductive and deductive in its thematic analysis and facilitates comparison across cases [21-23]. Results were shared with the LC to get their feedback and clarification.

\section{Results}

Table 1 presents basic characteristics of the full study sample alongside data about the subset of clients who 
Table 1 Characteristics of Larger Study Sample Alongside Characteristics of Clients Who Utilized RSN or CSP Services

\begin{tabular}{|c|c|c|c|c|c|c|}
\hline & \multicolumn{3}{|c|}{ Full Claims Data Sample $(N=4491)$} & \multicolumn{3}{|c|}{$\begin{array}{l}\text { Subset of clients who utilized RSN or CSP Services } \\
(N=1740)\end{array}$} \\
\hline & Intervention & TAU & $p$-value & RSN clients & CSP clients & $p$-value \\
\hline Number of clients & $2667(59.4 \%)$ & $1824(40.6 \%)$ & & $816(46.9 \%)$ & $924(53.1 \%)$ & \\
\hline Gender $^{a}$ & & & 0.00 & & & 0.05 \\
\hline Female & $34.8 \%$ & $28.6 \%$ & & $35.5 \%$ & $31.1 \%$ & \\
\hline Male & $65.2 \%$ & $71.4 \%$ & & $64.5 \%$ & $68.9 \%$ & \\
\hline Age & & & 0.00 & & & 0.00 \\
\hline $18-24$ & $13.7 \%$ & $8.4 \%$ & & $14.6 \%$ & $7.7 \%$ & \\
\hline $25-29$ & $20.5 \%$ & $15.8 \%$ & & $18.4 \%$ & $16.5 \%$ & \\
\hline $30-39$ & $30.2 \%$ & $30.3 \%$ & & $30.5 \%$ & $31.2 \%$ & \\
\hline $40+$ & $35.6 \%$ & $45.5 \%$ & & $36.5 \%$ & $44.6 \%$ & \\
\hline \multicolumn{7}{|l|}{ Prior detox past year } \\
\hline$\leq 90$ days & $51.6 \%$ & $53.0 \%$ & 0.35 & $51.5 \%$ & $56.4 \%$ & 0.03 \\
\hline \multicolumn{3}{|c|}{$\begin{array}{l}\text { Charlson Comorbidity Score }(0=\text { no comorbidity; higher score indicates more } \\
\text { comorbidity) }\end{array}$} & 0.00 & & & 0.00 \\
\hline 0 & $59.1 \%$ & $51.5 \%$ & & $60.7 \%$ & $48.6 \%$ & \\
\hline 1 & $25.0 \%$ & $25.7 \%$ & & $24.5 \%$ & $27.8 \%$ & \\
\hline 2 & $7.9 \%$ & $9.9 \%$ & & $7.6 \%$ & $11.0 \%$ & \\
\hline 3 & $2.6 \%$ & $3.7 \%$ & & $2.2 \%$ & $3.6 \%$ & \\
\hline $4+$ & $5.4 \%$ & $9.2 \%$ & & $5.0 \%$ & $9.0 \%$ & \\
\hline At least 1 Mental health diagnosis & $77.8 \%$ & $79.0 \%$ & 0.34 & $79.5 \%$ & $83.1 \%$ & 0.06 \\
\hline \multicolumn{2}{|l|}{ Medicaid Enrollment Category } & & 0.00 & & & 0.00 \\
\hline Disabled & $29.0 \%$ & $38.1 \%$ & & $35.3 \%$ & $42.4 \%$ & \\
\hline Non-disabled (TANF) & $22.7 \%$ & $17.5 \%$ & & $29.6 \%$ & $16.7 \%$ & \\
\hline Basic & $8.3 \%$ & $10.0 \%$ & & $7.8 \%$ & $9.7 \%$ & \\
\hline Essential & $40.0 \%$ & $34.4 \%$ & & $27.3 \%$ & $31.4 \%$ & \\
\hline \multicolumn{2}{|c|}{ Medicaid enrolled prior to index detox } & & 0.53 & & & 0.40 \\
\hline$\leq 1$ year & $18.9 \%$ & $18.1 \%$ & & $16.5 \%$ & $18.0 \%$ & \\
\hline$>1$ year & $81.1 \%$ & $81.9 \%$ & & $83.5 \%$ & $82.0 \%$ & \\
\hline
\end{tabular}

${ }^{a}$ Study intake form allowed respondents to identify as transgender or other gender

utilized RSN or CSP services. Thirty-one percent of intervention site clients utilized RSN services during the study, while $51 \%$ of TAU clients utilized CSP. Focusing on those that utilized support services, the data indicates significant differences between RSN and CSP clients. The RSN sample included proportionately more females, and had more clients ages 18 to 24 and fewer clients aged 40 and older. RSN clients were less likely to have had a detox admission within the past 90 days, had fewer comorbid conditions and were less likely to have disability as their Medicaid enrollment category.

Table 2 presents the total RSN and CSP costs and the average cost per member per month for clients who engaged with post-detox services. On average, RSN clients utilized services for a substantially longer time than CSP clients (4.5 versus 0.5 months). Some of this difference may be due to the case rate being active for 30 days from the last date of service. If we were to adjust the CSP time by adding 30 days to the last day of service, the average time in service would remain substantially different, 1.5 months for CSP clients versus 4.5 months for RSN clients. While the total cost per client over the study period was 53\% higher for RSN clients (\$1139) compared to CSP clients (\$746), the average cost per

Table 2 Total RSN and CSP Costs Incurred Any Time After Index Detox Admission Based on Claims Data

\begin{tabular}{lll}
\hline & RSN & CSP \\
\hline Number of Clients & 816 & 924 \\
Total amount Paid & $\$ 929,833$ & $\$ 689,578$ \\
Average Length of Stay (months) & 4.5 months & 0.5 months \\
Average Total Cost Per Member Per Month & $\$ 255$ & $\$ 1458$ \\
Average Total Cost Per Member & $\$ 1139$ & $\$ 746$ \\
\hline
\end{tabular}


member per month was significantly less ( $\$ 255$ versus \$1458). To better understand this difference, we assessed service use patterns over time.

Figure 1 presents the distribution of RSN and CSP service engagement by the proportion of clients that fell into different time frames based on 30-day increments. Contrasting patterns of engagement were observed. The overwhelming majority of CSP clients, $82.4 \%$, utilized the service for 30 days or less. In contrast, over half (57.9\%) of RSN clients utilized the service for more than 90 days and only $12.0 \%$ of clients utilized the service for 30 days or less.

Next, analyses focused on the type of RSN service provided, using RSN service data. Table 3 presents service contact and outreach information for 799 RSN clients. Service contacts could begin while the client was still at the detox and could be scheduled in advance, not requiring an outreach attempt for scheduling. Outreach attempts typically took place after a missed service attempt or after some time had passed without any type of regularly scheduled contact with the client. The data indicate that $97 \%$ of clients had a service contact after having enrolled. These clients averaged 6.5 contacts, with $64.6 \mathrm{~min}$ per contact on average.

Nearly half (45\%) of RSN clients had an outreach attempt, with an average of 6.3 attempts per client $(n=$ 2290). RSNs spent a total of $193.5 \mathrm{~h}$ conducting outreach, with the average outreach attempt lasting approximately 5 min. RSNs spent approximately $32 \mathrm{~min}$ on outreach for each client they attempted to contact. Among the 799 RSN clients, 3.4\% received outreach only, 54.7\% received service contacts only, and $41.9 \%$ received both outreach and service contacts (Table 4).

\section{Qualitative findings on the role and impact of provider level context}

Findings from the key informant interviews and LCs fell into three major themes: impact on RSN Role, feasibility: benefits, and feasibility: challenges, and were consistent across sites. Within these themes, sub-themes emerged related to services provided to clients, training and coaching, prior authorization, staffing and payment level (see Fig. 2). All themes arising from the data are reported below.

Respondents indicated that RSN and CSP staff shared a commitment to help clients engage in further treatment and believed that the detox facility was the ideal place to recruit clients for their service. Focusing on the context and experience of RSNs at the intervention sites, the shift to case rate billing represented a substantial change in procedures and processes. As such, buy-in among workers and their supervisors was crucial.

Within intervention sites, there was an early unexpected resistance to the case rate, which was originally set at $\$ 8.40$ per day. Though later debunked, many RSNs perceived this as the equivalent of being paid $\$ 1$ per hour. RSNs also struggled to reconcile productivity targets already established under the unit-of-service system with the new case rate system. This created fears that they might not be able to meet performance expectations, which could cost them their jobs.

Further, respondents noted that because providers serve clients with different insurance plans, and only MBHP adopted the new RSN service, many RSNs continued to provide CSP services to non-MBHP clients (i.e., clients not in the study), curtailing some of the intended benefits of the case rate (e.g., simplification of paperwork, no need to request authorizations). We were not able to measure how much CSP-related work with non-study clients impinged on RSN-related tasks, nor how CSP workload and related productivity targets impacted RSNs' ability to recruit new clients or continue work with existing clients. However, RSNs were frequently challenged by having to navigate both payment systems simultaneously, even as they became more comfortable with the case rate over time.

RSN staffing levels represented a constant challenge. At the outset of the study, providers staffed the RSN positions based on their experience staffing for CSP positions, with no formal cap on caseload size. Over the

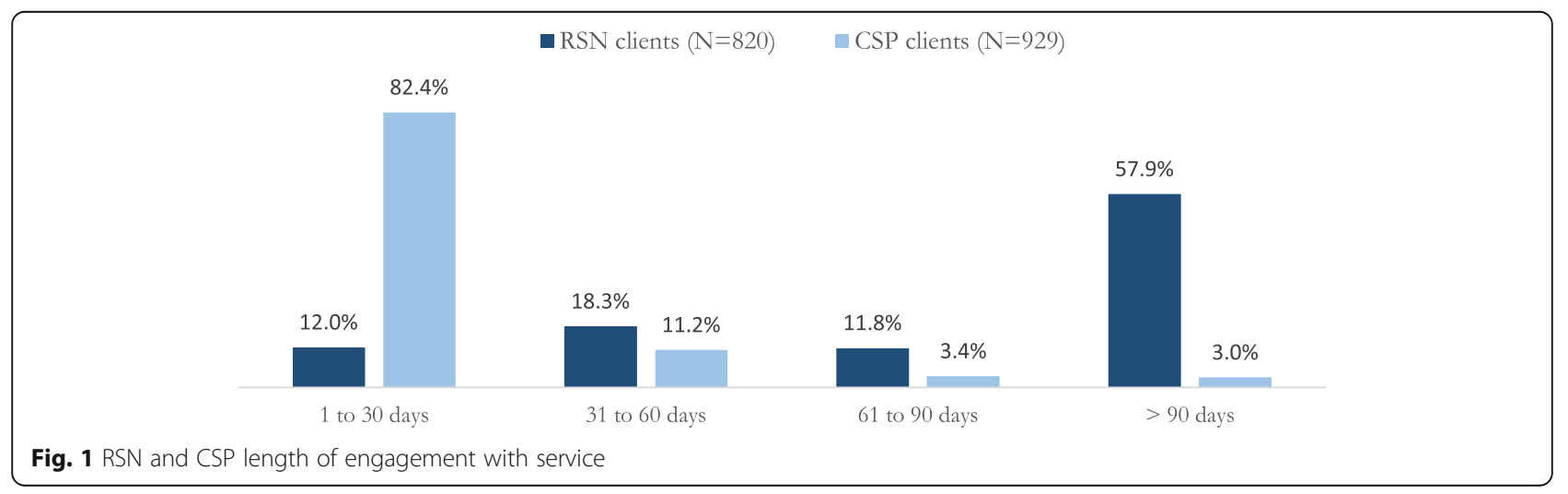


Table 3 RSN Service Delivery Data on Contacts and Outreach Attempts ( $\left.N=799^{a}\right)$

\begin{tabular}{|c|c|c|c|c|}
\hline & \multicolumn{2}{|c|}{ Service Contacts (excludes outreach) } & \multicolumn{2}{|c|}{ Outreach Attempts (excludes service) } \\
\hline & $\mathbf{N}$ & $\%$ & $\mathrm{~N}$ & $\%$ \\
\hline Clients with Service Contacts/Outreach Attempt & 772 & $97 \%$ & 362 & $45 \%$ \\
\hline Number of Service Contacts/Outreach Attempts & 5021 & & 2290 & \\
\hline Average Number of Contacts/Attempts per Member & 6.5 & & 6.3 & \\
\hline Time Spent: & Minutes & Hours & Minutes & Hours \\
\hline Total Time Spent & 324,286 & 5404.8 & 11,612 & 193.5 \\
\hline Average Time per Contact/Outreach Attempt & 64.6 & 1.1 & 5.1 & 0.08 \\
\hline Average Contact/Outreach Time per Member & 420.1 & 7.0 & 32.1 & 0.53 \\
\hline
\end{tabular}

${ }^{\mathrm{a}} \mathrm{RSN}$ service data were not available for $17 \mathrm{RSN}$ clients

course of the study, providers varied widely in terms of the caseload each RSN was expected to carry. This variation impacted the number of RSNs hired and the ability of RSNs to provide care to their clients. Additionally, providers hesitated to add new RSNs until they were certain new staff would have an appropriate minimum caseload. This resulted in caseload fluctuations throughout the project. Although the number of CSP staff also varied over time, this was less of an issue for providers that paid staff FFS.

Despite these challenges, positive impacts of the case rate were reported. When discussing the impact of case rate billing on service delivery, RSNs and their supervisors frequently highlighted how the case rate increased RSNs' ability to access group trainings or coaching activities that they would have struggled to attend under the FFS system. Moreover, RSNs valued the unfettered ability to conduct outreach to connect with clients who might be struggling with sustaining motivation for treatment. RSNs felt this level of outreach helped them retain clients in services longer, as they could reconnect with clients who had not been in contact for a while. Finally, they appreciated the reduction in administrative burden resulting from the case rate payment method, compared to billing FFS.

\section{Discussion}

Using the MRC framework to assess feasibility of case rate billing, we focused on whether case rate billing offered an economical alternative to FFS billing, whether the case rate payment method improved client length of

Table 4 RSN Service: Types of Contacts for RSN Clients

\begin{tabular}{lll}
\hline Contact Type & N & $\%$ \\
\hline Outreach Only & 27 & $3.4 \%$ \\
Service Only & \\
Both Outreach and Service & 437 & $54.7 \%$ \\
TOTAL & 335 & $41.9 \%$ \\
\hline
\end{tabular}

${ }^{\mathrm{a} S e r v i c e}$ only contacts indicate contacts that did not require an outreach attempt for the service to take place

${ }^{\mathrm{b}} \mathrm{RSN}$ service data were not available for $17 \mathrm{RSN}$ clients engagement for clients receiving RSN services compared to clients receiving CSP services paid FFS, and how advantages and disadvantages of the case rate payment method influenced provider practices. Another consideration is whether and how the case rate advanced or supported the CMS' triple aims of healthcare: reducing per capita costs, improving population health (increasing access), and improving the individual's experience of care (quality) [24].

Our analysis indicates that the total cost of RSN services paid via the case rate was approximately 50\% higher than for clients using CSP services paid FFS. At first blush, it appears the case rate failed the first test of feasibility in terms of offering an economical alternative to FFS billing. However, the answer is much more nuanced. RSN clients engaged with the service over a significantly longer period of time, resulting in a lower per member per month cost. Further, longer engagement has been shown to impact the quantity and quality of community-based services utilized by clients [25]. Analysis of the full claims data sample assessing service use and health care costs supports this assertion, showing increased rates of initiation with SUD treatment [26] and a shift in service use from more to less acute settings for the intervention group sample compared to TAU [18]. It also showed a slower rate of growth in health care spending for intervention group members than for TAU [18].

In terms of provider experience, simplicity of billing under a case rate payment model was an advantage. Case rate billing relieves administrative burden for providers, a benefit that also extends to payers. However, organizations and staff can only fully realize the administrative benefits of case rate payment if it is adopted by all or most payers. As noted earlier, the case rate improves engagement within the service by allowing for outreach not covered under FFS billing, and improves quality by creating opportunities for RSNs to participate in on-going coaching and training activities without consequence to their income or productivity targets. 


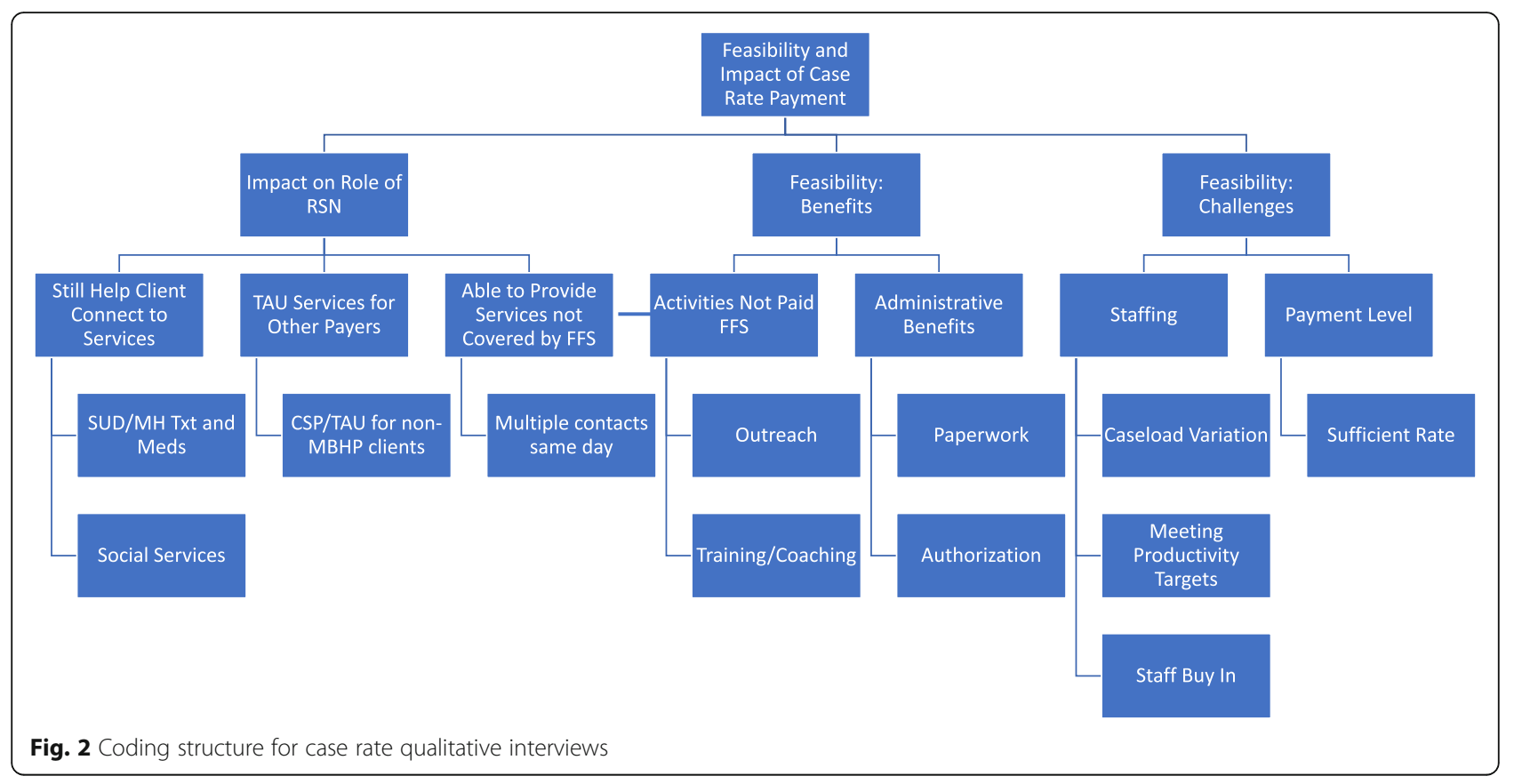

Finally, staff faced challenges implementing the case rate approach. First, staff had to re-conceptualize how they used their time and monitored productivity. Second, staffing levels for RSNs caseloads fluctuated throughout the study. If caseload counts were very high or if they included very complex clients that required more time, clients may have had to wait longer for services, reducing access and possibly artificially extending the duration of service. Yet, any such delay would be limited by the case rate requirement that RSNs have contact with clients at least once every 30 days.

Supporting the argument for feasibility, case rate billing supported longer engagement with services, which can increase access to needed care while also improving overall health [27]. The case rate also facilitated RSN investment in training, coaching and education, supporting higher quality care. Of greatest importance, longer engagement and increased connections to community-based services produces cost savings across systems of care by connecting individuals with appropriate community-based care and reducing inappropriate or costly emergency room visits [18]. However, it is unclear how a payer, like MBHP, would be able to recoup any savings that occurred outside behavioral health.

At the conclusion of the study, MBHP continued implementing case rate billing for RSN services. Effective July 2018, MassHealth added RSN services as a covered benefit, including MBHP members, but it chose to pay using unit rates rather than case rates. This study demonstrates that case rates offer an alternative payment approach that can benefit both providers and consumers.
However, until we see broader adoption across payers, providers may not realize the full benefits of case rate payments.

\section{Limitations}

Despite our large sample size and the depth of the data available for analysis, the study had some limitations. This intervention contained many interacting components and changes in the payment approach may have coincided with other changes, thereby influencing our findings. For example, the trainings administered to RSN workers could have impacted practice patterns and RSNs' abilities to engage with clients. Therefore, some of the changes observed may result from training and not just the case rate. On the other hand, the case rate payment structure made it easier for RSNs to fully participate in the trainings.

This study built on an existing workforce and service. As such, variation in the implementation, utilization and management of RSN or CSP services across providers existed before the study and the study did not require them to make any changes to participate. It is unknown whether or how these variations impacted uptake of either service. The intervention was at the site level and study subjects' treatment assignments were not randomized, reducing potential for causal inferences. Lastly, the quantitative analysis did not control for differences across sites nor group assignment in the analysis. We did not control for these differences because we were not running predictive models. We were assessing the feasibility of the case rate and, thus, looking at actual units billed and paid for under the case rate. However, 
we recognize that site and group differences could have impacted the results.

\section{Conclusion}

Assessing the overall contribution of the case rate in relation to outcomes we found that case rate billing could be a feasible alternative to FFS billing for RSN/CSP services for this population; although there are important caveats to consider.

Our findings highlight the ways in which case rate payment provides value and facilitates successful client engagement. However, when we focus on the providerlevel context, issues based on the variation in payment model for RSN versus CSP services rise to the surface. Most providers work with multiple payers. If only one payer offers this model of payment for a widely used service, any accrued benefits may be minimized or lost.

Despite growing emphasis on healthcare payment reform, which involves episode, global or other capitated payments from payers to healthcare systems [28, 29], payments from healthcare systems to providers typically remain FFS. If adopted broadly, case rates may offer an opportunity to increase client engagement and quality of care while reducing overall healthcare costs.

\section{Supplementary Information}

The online version contains supplementary material available at https://doi. org/10.1186/s12913-020-05861-8.

Additional file 1: Table S1a. Characteristics of Larger Study Sample by Programs - Full Claims Data Sample $(N=4,491)$. Table S1b.

Characteristics of Clients Who Utilized RSN or CSP Services by Programs Subset of clients who utilized RSN or CSP Services $(N=1,740)$.

\section{Abbreviations \\ CMS: Centers for Medicare and Medicaid Services; CSP: Community Support Program staff; FFS: Fee-for-Service billing; ICD-9: International Classification of Diseases, Ninth Revision; LC: Learning Collaborative; MBHP: Massachusetts Behavioral Health Partnership; MRC: Medical Research Council; PCC: Primary Care Clinician; RSN: Recovery Support Navigator; SUD: Substance Use Disorder; TAU: Treatment-as-Usual}

\section{Acknowledgements}

We would like to thank our CMS Project Officer, Sam Ortiz, who was a strong partner in implementing the best research possible within clinical settings. We would also like to thank the detoxification provider organizations that participated in the study and their CSP and RSN supervisors and staff. We thank our partners at MBHP who made this study possible including Scott Taberner, Nancy Norman, Ashley Wilson, Oliver Lyons, Barbara Mondragon and Jim Clarkson. At Brandeis, we thank Deborah Garnick, Michele Hutcheon, Wanda Rifkin, Amity Quinn, Jennifer Miles, and Galina Zolotusky for their support.

\section{Authors' contributions}

Drs. Torres and Brolin were involved in study design, data collection and analysis, and interpretation of results. Together, they drafted the paper, incorporated feedback from co-authors who offered substantive comments and revisions to the drafts, and finalized the paper for submission. Mr. Panas and Dr. Ritter were involved in study design, analyses and interpretation of results and reviewed drafts of the paper and provided substantial feedback. Drs. Merrick, Horgan and Lane conceived the research project and were involved in study design, analysis and interpretation of results. They contributed to the writing and revision of the manuscript. Drs. Hodgkin and Lee and Ms. Hopwood, Ms. Gewirtz and Ms. De Marco contributed to study design, data collection and analysis and interpretation of results. They also provided revisions to the drafts of the paper. All the authors read and approved the final manuscript.

\section{Funding}

This study was supported by Grant Number 1C1CMS331059-01-00 from the Department of Health and Human Services, Centers for Medicare \& Medicaid Services. The contents of this publication are solely the responsibility of the authors and do not necessarily represent the official views of the U.S. Department of Health and Human Services or any of its agencies. The research presented here was conducted by the awardee. Findings might or might not be consistent with or confirmed by the independent evaluation contractor.

\section{Availability of data and materials}

The data and materials used for this sub-study are from the state Medicaid provider and are not publicly available.

\section{Ethics approval and consent to participate}

The study protocol was reviewed and approved by the New England Institutional Review Board. Secondary data for claims and RSN log data did not require client consent. Meeting summaries for the Learning Collaboratives also did not require consent. Individuals taking part in key informant interviews signed a written consent prior to the interview taking place.

\section{Consent for publication}

Not applicable.

\section{Competing interests}

The authors declare that they have no competing interests.

\section{Author details}

${ }^{1}$ Brandeis University, Heller School for Social Policy and Management, Waltham, MA 02453, USA. ${ }^{2}$ Smith College School for Social Work, Lilly Hall, Northampton, MA 01060, USA. ${ }^{3}$ Massachusetts Behavioral Health Partnership, a Beacon Health Options company, 1000 Washington Street, Suite 310, Boston, MA 02118, USA. ${ }^{4}$ Harvard Medical School and Harvard Pilgrim Health Care Institute, 401 Park Drive, Suite 401 East, Boston, MA 02215, USA.

Received: 3 February 2020 Accepted: 25 October 2020

Published online: 03 November 2020

\section{References}

1. Mark TL, Vandivort-Warren R, Montejano LB. Factors affecting detoxification readmission: analysis of public sector data from three states. J Subst Abus Treat. 2006:31(4):439-45.

2. Carrier E, McNeely J, Lobach I, Tay S, Gourevitch MN, Raven MC. Factors associated with frequent utilization of crisis substance use detoxification services. J Addict Dis. 2011;30(2):116-22.

3. Amodeo M, Lundgren L, Chassler D, Witas J. High-frequency users of detoxification: who are they? Subst Use Misuse. 2008;43(7):839-49.

4. Spear SE. Reducing readmissions to detoxification: an interorganizational network perspective. Drug Alcohol Depend. 2014;137:76-82.

5. Neighbors CJ, Yemeni R, O'Grady MA, Sun Y, Morgenstern J. Recurrent use of inpatient withdrawal management services: characteristics, service use, and cost among Medicaid clients. J Subst Abus Treat. 2018;92:77-84.

6. MBHP. Personal communication; 2012.

7. Quinn AE, Hodgkin D, Perloff JN, Stewart MT, Brolin M, Lane N, et al. Design and impact of bundled payment for detox and follow-up care. J Subst Abus Treat. 2017;82:113-21.

8. Padwa H, Urada D, Gauthier P, Rieckmann T, Hurley B, Crèvecouer-MacPhail D. Organizing publicly funded substance use disorder treatment in the United States: moving toward a service system approach. J Subst Abus Treat. 2016;69:9.

9. Garnick DW, Lee MT, Horgan CM, Acevedo A. Adapting Washington circle performance measures for public sector substance abuse treatment systems. J Subst Abus Treat. 2009;36(3):265-77. 
10. Performance Specifications - Community Support Program (CSP) [https:// www.masspartnership.com/pdf/CSPFINALul2014.pdf].

11. Alert \#22: 15-Minute Unite Billing Clarification [https://www.masspartnership. com/pdf/HNEAlert22_15minuteUnitsFIN11513.pdf].

12. Miller HD. Why value-based payment Isn't working, and how to fix it: creating a patient-centered payment system to support higher-quality, more affordable health carehttp://www.chqpr.org/reports.html: Center for Healthcare Quality \& Payment Reform; 2017.

13. Miller HD. From volume to value: better ways to pay for health care. Health Aff. 2009;28(5):1418-28.

14. Center for Healthcare Quality \& Payment Reform: The Payment Reform Glossary. In. http://www.chqpr.org/reports.html.

15. de Brantes F, Rosenthal MB, Painter M. Building a bridge from fragmentation to accountability — the Prometheus payment model. N Engl J Med. 2009;361(11):1033-6.

16. Moore GF, Audrey S, Barker M, Bond L, Bonell C, Hardeman W, et al. Process evaluation of complex interventions: Medical Research Council guidance. BMJ. 2015;350:h1258.

17. Craig P, Dieppe P, Macintyre S, Michie S, Nazareth I, Petticrew M. Developing and evaluating complex interventions: the new Medical Research Council guidance. BMJ. 2008;337:587.

18. Hodgkin D, Brolin M, Ritter G, Torres M, Merrick E, Horgan C, et al. Cost savings from a navigator intervention for repeat detoxification clients. J Ment Health Policy Econ. 2019;22:3-13.

19. Brolin M, Torres M, Hodgkin D, Horgan C, Lee M, Merrick E, et al. Implementation of client incentives within a recovery navigation program. J Subst Abus Treat. 2017;72:25-31.

20. Charlson ME, Pompei P, Ales KL, Mackenzie CR. A new method of classifying prognostic comorbidity in longitudinal studies: development and validation. J Chronic Dis. 1987;40(5):373-83.

21. Gale NK, Heath G, Cameron E, Rashid S, Redwood S. Using the framework method for the analysis of qualitative data in multi-disciplinary health research. BMC Med Res Methodol. 2013;13:117.

22. Smith J, Firth J. Qualitative data analysis: the framework approach. Nurs Res. 2011;18(2):52-62.

23. Pope C, Ziebland S, Mays N. Qualitative research in health care. Analysing qualitative data. BMJ. 2000;320(7227):114-6.

24. Berwick DM, Nolan TW, Whittington J. The triple aim: care, health, and cost. Health Aff (Millwood). 2008;27(3):759-69.

25. Schaefer JA, Ingudomnukul E, Harris AH, Cronkite RC. Continuity of care practices and substance use disorder patients' engagement in continuing care. Med Care. 2005;34:1234-41.

26. Lee MT, Torres M, Brolin M, Merrick E, Ritter G, Panas L, et al. Impact of recovery support navigators on continuity of care after detoxification. J Subst Abus Treat. 2020;112:10-6.

27. Schulte MT, Hser Y-I. Substance use and associated health conditions throughout the lifespan. Public Health Rev. 2013;35:2.

28. Mechanic R, Altman S. Payment reform options: episode payment is a good place to start. Health Affairs (Project Hope). 2009;28(2):W262-71.

29. Korda $H$, Eldridge G. Payment incetnives and integrated care delivery: levers for health system reform and cost containment. J Health Care Organ Prov Financ. 2011;48(4):277-87.

\section{Publisher's Note}

Springer Nature remains neutral with regard to jurisdictional claims in published maps and institutional affiliations.

Ready to submit your research? Choose BMC and benefit from:

- fast, convenient online submission

- thorough peer review by experienced researchers in your field

- rapid publication on acceptance

- support for research data, including large and complex data types

- gold Open Access which fosters wider collaboration and increased citations

- maximum visibility for your research: over $100 \mathrm{M}$ website views per year

At BMC, research is always in progress.

Learn more biomedcentral.com/submissions 